\title{
Representations of Linear Dual Rate System via Single SISO LTI Filter, Conventional Sampler and Block Sampler
}

\author{
C. Y. F. Ho ${ }^{1}$, Student Member, IEEE, B. W. K. Ling ${ }^{2}$, and P. K. S. Tam ${ }^{3}$, Member, IEEE
}

\begin{abstract}
In this brief, it is proved that a linear dual rate system can be represented via a series cascade of (i) a conventional expander, a single input single output (SISO) linear time invariant (LTI) filter and a block decimator, or (ii) a block expander, an SISO LTI filter and a conventional decimator. Hence, incompatible nonuniform filter banks could achieve perfect reconstruction via LTI filters, conventional samplers and block samplers without expanding the input output dimension of a subsystem of linear dual rate systems or converting the nonuniform filter banks to uniform filter banks. The main advantage of the proposed representations is to avoid complicated design of the circuit layout caused by connecting subsystems with large input output dimension or a lot of subsystems together.
\end{abstract}

Index Terms - Incompatible, nonuniform filter bank, direct representation, perfect reconstruction.

\section{INTRODUCTION}

$\mathrm{N}$ ONUNIFORM filter banks are important not only because they are widely employed in audio, radar and transmultiplexing applications, but they could achieve more general time and frequency tiling in which conventional tree structured two channel filter banks could not [1], [2].

However, there is a kind of nonuniform filter banks, called incompatible nonuniform filter banks [3], which do not achieve perfect reconstruction via non-ideal LTI filters and conventional samplers (without expanding the channels) [3]. There are two common approaches for tackling the perfect reconstruction problem. The first approach is to convert the nonuniform filter banks to uniform filter banks [3]. This approach is regarded as an indirect approach. Although the total number of filter coefficients of the corresponding uniform filter banks is the same as that of filter banks we

Manuscript received June 18, 2007; revised July 30, 2007. This work was substantially supported by a research grant (project number G-YD26) from the Hong Kong Polytechnic University, the Centre for Multimedia Signal Processing, The Hong Kong Polytechnic University, the CRGC grant (project number PolyU 5101\01E) from the Research Grants Council of Hong Kong.

Bingo Wing-Kuen Ling is with the department of Electronic Engineering, King's College London (e-mail: wing-kuen.ling@kcl.ac.uk). Charlotte YukFan Ho is with the department of Electronic Engineering, Queen Mary, University of London. Peter Kwong-Shun Tam is with the department of Electronic and Information Engineering, The Hong Kong Polytechnic University.

Copyright (c) 2007 IEEE. Personal use of this material is permitted. However, permission to use this material for any other purposes must be obtained from the IEEE by sending an email to pubs-permission@ieee.org. propose via direct approaches in this brief, the number of channels of the corresponding uniform filter banks is equal to the least common multiple (LCM) of the set of decimation integers of the nonuniform filter banks. Sometimes, the LCM of the set of decimation integers could be very large. For example, for this set of decimation integers $\{2,5,7,10,35,35\}$, the LCM is 70 . This implies that 70 channels are required for the implementation of the corresponding uniform filter bank. Compared to the original 6 channel nonuniform filter bank, the design of the circuit layout of the corresponding uniform filter bank is more complicated because more filters and samplers are required to be connected together. For the second approach, the LTI filters and the conventional samplers are generalized to linear dual rate systems [4]. Linear dual rate systems are linear systems for which shifting an input by $n$ samples resulting in shifting an output by $m$ samples. There are three common realizations for linear dual rate systems. The first realization is to represent a linear dual rate system via a linear time periodic varying (LTPV) subsystem and a set of block samplers. For this realization, the input output relationship of the LTPV subsystem is no longer governed by the multiplication of its input and its frequency response. However, the fundamental working principle of filter banks is to decompose an input signal into different frequency bands and different processing units are tailored based on the characteristics of the decomposed subband signals. Hence, this realization would cause difficulties for the design of the subband processing units. The second realization is to represent a linear dual rate system via a vector sample rate changer. The dimension of the LTI filter in the vector sample rate changer depends on the highest common factor (HCF) of $m$ and $n$, where $m$ and $n$ are defined as above. The last realization is to represent a linear dual rate system via a linear switching time varying subsystem and a set of conventional samplers. The linear switching time varying subsystem is further realized via a parallel connection of LTI filters and a set of conventional samplers. The number of LTI filters required for the realization is also equal to the HCF of $m$ and $n$, where $m$ and $n$ are defined as above. For the second and the third realizations, although LTI filters are employed, the input output dimension of the subsystem required for the representations is expanded and equal to the HCF of $m$ and $n$. Similar to the indirect approach, sometimes the HCF of $m$ and $n$ may be very large and these two approaches require complicated design of the circuit layout. 
The outline of this brief is as follows. Notations and definitions used throughout this brief are introduced in Section II. In Section III, a linear dual rate system is proved to be possible to be represented via a series cascade of (i) a conventional expander, an SISO LTI filter and a block decimator, or (ii) a block expander, an SISO LTI filter and a conventional decimator. In Section IV, an incompatible nonuniform filter bank is shown to achieve perfect reconstruction via LTI filters, conventional samplers and block samplers. Finally, a conclusion is drawn in Section V.

\section{NOTATIONS AND DEFINITIONS}

Denote $N$ as the number of channels of a nonuniform filter bank. Denote $H_{i}(z)$ and $F_{i}(z)$ for $i=0,1, \cdots, N-1$ as respectively, the analysis and synthesis filters, where $H_{i}(z)$ and $F_{i}(z)$ for $i=0,1, \cdots, N-1$ are assumed to be LTI filters. A conventional decimator with a decimation integer $p_{i}$ and a conventional expander with an expansion integer $p_{i}$ are denoted as, respectively, $\downarrow p_{i}$ and $\uparrow p_{i}$. A block decimator with a block length $q$ and a decimation integer $p_{i}$, and a block expander with a block length $q$ and an expansion integer $p_{i}$ are denoted as, respectively, $\downarrow\left(p_{i}, q\right)$ and $\uparrow\left(p_{i}, q\right)$. It is worth noting that when $q$ is equal to 1 , the block samplers reduce to the conventional samplers [5], [6].

For a maximally decimated nonuniform filter bank with decimation integers $p_{i}$ for $i=0,1, \cdots, N-1$, denote $l_{i}$ as an integer such that $l_{i} \in\left\{0,1, \cdots, p_{i}-1\right\}$. If for every $p_{i}$ and $l_{i} \in\left\{0,1, \cdots, p_{i}-1\right\}$, there exists $p_{j}$ and $l_{j} \in\left\{0,1, \cdots, p_{j}-1\right\}$ such that $i \neq j$ and $\frac{l_{i}}{p_{i}}=\frac{l_{j}}{p_{j}}$, then the nonuniform filter bank is called a compatible nonuniform filter bank. Otherwise, it is called an incompatible nonuniform filter bank [3].

\section{REPRESENTATIONS OF A LINEAR DUAL RATE SYSTEM VIA AN SISO LTI FILTER, A CONVENTIONAL SAMPLER AND A BLOCK SAMPLER}

\section{A. Motivation}

A linear dual rate system can always be represented via a kernel function $\mathrm{g}$ such that

$$
\mathrm{y}[k]=\sum_{l \rightarrow-\infty}^{+\infty} \mathrm{g}[k, l] \mathrm{u}[l], \forall k \in \mathrm{Z},
$$

where $u[l]$ and $y[k]$ are the input and the output of the linear dual rate system, respectively. Since linear dual rate systems are defined as shifting an input by $n$ samples resulting in shifting an output by $m$ samples, we have

$$
\mathrm{g}[k, l]=\mathrm{g}[k+m, l+n], \forall k, l \in \mathrm{Z} .
$$

For a conventional $m / n$ rate changer with $\uparrow m$, an LTI filter with an impulse response f[k] and $\downarrow n$ [4], the input output relationship is:

$$
\mathrm{y}[\mathrm{k}]=\sum_{l \rightarrow-\infty}^{+\infty} \mathrm{f}[k n-\operatorname{lm}] \mathrm{u}[l], \forall k \in \mathrm{Z} .
$$

If the linear dual rate system can be represented via the conventional $\mathrm{m} / \mathrm{n}$ rate changer, then the transformation between $g[k, l]$ and $\mathrm{f}[l]$ is:

$$
\mathrm{f}[k n-l m]=\mathrm{g}[k, l], \forall k, l \in \mathrm{Z} .
$$

However, according to the Euclid's theorem, the mapping from $\{0,1, \cdots, m-1\} \times \mathrm{Z}$ to $\mathrm{Z}$, where $[k, l] \in\{0,1, \cdots, m-1\} \times \mathrm{Z}$ and $k n-l m \in Z$, is bijective if and only if $m$ and $n$ are co-prime. Hence, linear dual rate systems can be realized via conventional $\mathrm{m} / \mathrm{n}$ rate changers if and only if $m$ and $n$ are coprime [4].

The mapping between $\mathrm{g}[k, l]$ and $\mathrm{f}[k]$ is illustrated in Figure 1. The $x$-axis and the $y$-axis of Figure 1 are the time indices $l$ and $k$, respectively. The value located at $(l, k)$ is $g[k, l]$. Since for a given time index $(l, k)$, it corresponds to a unique time index $k n-l m$. Hence, $\mathrm{g}[k, l]$ also corresponds to a unique value of $\mathrm{f}[k n-l m]$. Figure 1 shows the values of $\mathrm{f}[\mathrm{kn}-\mathrm{Im}]$ at different time indices $(l, k)$. Figure 1a shows the case when $m$ and $n$ are co-prime. Since linear dual rate systems satisfy (2), shifting Figure 1a by $n$ samples horizontally and $m$ samples vertically will correspond to the same figure. It can be seen from Figure 1a that $\mathrm{f}[k n-l m]$ is periodic with a horizontal period $n$ and a vertical period $m$, and the conventional $m / n$ rate changer is $(m, n)$ shift invariant. Figure $1 \mathrm{~b}$ shows the case when $n$ is an integer multiple of $m$ with the factor $c$, that is $n=c m$. When the time index $(l, k)$ is shifted horizontally by $c$ and vertically by $1, l$ becomes $l+c$ and $k$ becomes $k+1$. Hence, $k n-l m$ becomes $(k+1) n-(l+c) m$, which is equal to $k n-l m$ because $n=c m$. Consequently, $\mathrm{f}[k n-l m]$ has a horizontal period $c$ and a vertical period 1 , and the conventional $\mathrm{m} / \mathrm{n}$ rate changer is $(1, c)$ shift invariant, instead of $(\mathrm{m}, \mathrm{cm})$ shift invariant. This implies that the conventional $m / n$ rate changer cannot represent a general $(m, c m)$ shift invariant linear dual rate system. Figure 1c shows the case when $m$ is an integer multiple of $n$ with the factor $c$, that is $m=c n$. When the time index $(l, k)$ is shifted horizontally by 1 and vertically by $c, l$ becomes $l+1$ and $k$ becomes $k+c$. Hence, $k n-l m$ becomes $(k+c) n-(l+1) m$, which is equal to $k n-l m$ because $m=c n$. Consequently, $\mathrm{f}[k n-l m]$ has a horizontal period 1 and a vertical period $c$, and the conventional $\mathrm{m} / \mathrm{n}$ rate changer is $(c, 1)$ shift invariant, instead of $(c n, n)$ shift invariant. This implies that the conventional $m / n$ rate changer cannot represent a general $(c n, n)$ shift invariant linear dual rate system. Figure $1 \mathrm{~d}$ shows the case when $m=n$. When the time index $(l, k)$ is shifted both horizontally and vertically by $1, l$ becomes $l+1$ and $k$ becomes $k+1$. Hence, $k n-l m$ becomes $(k+1) n-(l+1) m$, which is equal to $k n-l m$ because $m=n$. Consequently, $\mathrm{f}[k n-l m]$ has the trivial horizontal period 1 and vertical period 1 , and the conventional $m / n$ rate changer is $(1,1)$ shift invariant, instead of $(n, n)$ shift invariant, or $n$ time periodic varying. This implies that the conventional $\mathrm{m} / \mathrm{n}$ rate changer 
cannot represent a general $(n, n)$ shift invariant linear dual rate system.

\section{B. First proposed representation}

\section{Theorem 1}

$\forall m, n \in \mathrm{Z}^{+}$(no matter $m$ and $n$ are co-prime or not), all linear dual rate systems can be represented via a series cascade of $\uparrow m$, followed by an LTI filter with an impulse response $\mathrm{f}[k]$, and then followed by $\downarrow(n, m)$.

Proof: is:

The input output relationship of all linear dual rate systems

$\mathrm{y}[k m+i]=\sum_{l \rightarrow-\infty}^{+\infty}[i, l-k n] \mathrm{u}[l], \forall k, l \in \mathrm{Z}, \forall m, n \in \mathrm{Z}^{+}$and $i=0,1, \cdots, m-1$.

On the other hand, the input output relationship of the proposed representation is:

$\mathrm{y}[k m+i]=\sum_{\forall l} \mathrm{f}[k m n-m l+i] \mathrm{u}[l], \forall k, l \in \mathrm{Z}, \forall m, n \in \mathrm{Z}^{+}$and $i=0,1, \cdots, m-1$.

It is shown in Appendix A that $\forall k, l \in Z, \forall m, n \in Z^{+}$and $i=0,1, \cdots, m-1$, the mapping from $\{0,1, \cdots, m-1\} \times Z$ to $Z$, where $[i, l-k n] \in\{0,1, \cdots, m-1\} \times Z$ and $k m n-m l+i \in Z$, is bijective. Hence, $\forall k, l \in Z, \forall m, n \in Z^{+}$and $i=0,1, \cdots, m-1$, there exists a unique time index $k m n-m l+i$ corresponding to the time index $[i, l-k n]$. As a result, there exists an LTI filter with an impulse response $f[k]$ satisfying:

$\mathrm{f}[k m n-m l+i]=\mathrm{g}[i, l-k n], \forall k, l \in \mathrm{Z}, \forall m, n \in \mathrm{Z}^{+}$and $i=0,1, \cdots, m-1,(7)$ that the linear dual rate systems and the proposed representation are input output equivalent. This completes the proof.

\section{C.Second proposed representation}

Theorem 2

$\forall m, n \in \mathrm{Z}^{+}$(no matter $m$ and $n$ are co-prime or not), all linear dual rate systems can be represented via a series cascade of $\uparrow(m, n)$, followed by an LTI filter with an impulse response $\mathrm{f}[k]$, and then followed by $\downarrow n$.

Proof:

The input output relationship of the proposed representation is:

$\mathrm{y}[k]=\sum^{+\infty} \sum^{n-1} \mathrm{f}[k n-m n l-i] \mathrm{u}[n l+i], \forall k, l \in \mathrm{Z}, \forall m, n \in \mathrm{Z}^{+}$and $i=0,1, \cdots, n-1$.

On the other hand, the input output relationship of linear dual rate systems is:

$$
\mathrm{y}[k]=\sum_{l \rightarrow-\infty}^{+\infty} \sum_{i=0}^{n-1} \mathrm{~g}[k, n l+i] \mathrm{u}[n l+i], \forall k, l \in \mathrm{Z}, \forall m, n \in \mathrm{Z}^{+} \text {and } i=0,1, \cdots, n-1 .
$$

It is shown in Appendix $\mathrm{B}$ that $\forall l \in \mathrm{Z}, \quad \forall m, n \in \mathrm{Z}^{+}$, $k \in\{0,1, \cdots, m-1\}$ and $i \in\{0,1, \cdots, n-1\}$, the mapping from $\{0,1, \cdots, m-1\} \times \mathrm{Z}$ to $\mathrm{Z}$, where $[k, n l+i] \in\{0,1, \cdots, m-1\} \times \mathrm{Z}$ and $k n-m n l-i \in Z$, is bijective. Since (2) is satisfied, $\forall l \in Z$, $\forall m, n \in \mathrm{Z}^{+}, k \in\{0,1, \cdots, m-1\}$ and $i \in\{0,1, \cdots, n-1\}$, there exists a unique time index $k n-m n l-i$ corresponding to the time index $[k, n l+i]$. As a result, there exists an LTI filter with an impulse response $\mathrm{f}[k]$ satisfying: $\mathrm{f}[k n-m n l-i]=\mathrm{g}[k, n l+i], \forall k, l \in \mathrm{Z}, \forall m, n \in \mathrm{Z}^{+}$and $i=0,1, \cdots, n-1$, that the proposed representation and the linear dual rate systems are input output equivalent. This completes the proof.

\section{D.Discussions and verifications}

It is worth noting that one of the existing realizations of linear dual rate systems also involves block samplers, but it still requires an LTPV subsystem with its periodicity equal to the HCF of $m$ and $n$. On the other hand, our proposed realizations do not involve LTPV subsystem. This is because the symmetric representation of the existing realization cannot cancel the HCF of $m$ and $n$. On the other hand, our proposed representations are asymmetric so that they can cancel the HCF of $m$ and $n$. Therefore, we can simply employ an LTI filter, which is generally simpler than the LTPV or linear switching time varying counterparts, as its impulse and frequency responses are more easily exploited.

To verify our proposed representations, the conventional samplers and the block samplers are represented via matrix forms because they are both linear systems. Let $\mathbf{I}_{i, j}$ be an $i$ by $j$ matrix with its upper rightmost element being 1 and 0 elsewhere. Similarly, let $\mathbf{I}_{i, j}^{\prime}$ be an $i$ by $j$ matrix with diagonal elements equal to 1 and 0 elsewhere. $\uparrow m$ can be represented as:

$$
\left[\begin{array}{c}
\vdots \\
\mathrm{y}[0] \\
\mathrm{y}[1] \\
\vdots
\end{array}\right]=\left[\begin{array}{cccc}
\ddots & \ddots & & \\
\ddots & \mathbf{I}_{m, 1} & \mathbf{0} & \\
& \mathbf{0} & \mathbf{I}_{m, 1} & \ddots \\
& & \ddots & \ddots
\end{array}\right]\left[\begin{array}{c}
\vdots \\
\mathrm{u}[0] \\
\mathrm{u}[1] \\
\vdots
\end{array}\right]
$$

while $\downarrow(n, m)$ can be represented as:

$$
\left[\begin{array}{c}
\vdots \\
\mathrm{y}[0] \\
\mathrm{y}[1] \\
\vdots
\end{array}\right]=\left[\begin{array}{cccc}
\ddots & \ddots & & \\
\ddots & \mathbf{I}_{m, n m}^{\prime} & \mathbf{0} & \\
& \mathbf{0} & \mathbf{I}_{m, n m}^{\prime} & \ddots \\
& & \ddots & \ddots
\end{array}\right]\left[\begin{array}{c}
\vdots \\
\mathrm{u}[0] \\
\mathrm{u}[1] \\
\vdots
\end{array}\right] .
$$

The input output relationship of our first proposed representation is:

$$
\left[\begin{array}{c}
\vdots \\
\mathrm{y}[0] \\
\mathrm{y}[1] \\
\vdots
\end{array}\right]=\left[\begin{array}{cccc}
\ddots & \ddots & & \\
\ddots & \mathbf{I}_{m, n m}^{\prime} & \mathbf{0} & \\
& \mathbf{0} & \mathbf{I}_{m, n m}^{\prime} & \ddots \\
& & \ddots & \ddots
\end{array}\right]\left[\begin{array}{cccc}
\ddots & \vdots & \vdots & \cdot \\
\cdots & \mathrm{f}[1] & \mathrm{f}[0] & \cdots \\
\cdots & \mathrm{f}[2] & \mathrm{f}[1] & \cdots \\
\ddots & \vdots & \vdots & \ddots
\end{array}\right]\left[\begin{array}{cccc}
\ddots & \ddots & & \\
\ddots & \mathbf{I}_{m, 1} & \mathbf{0} & \\
& \mathbf{0} & \mathbf{I}_{m, 1} & \ddots \\
& & \ddots & \ddots
\end{array}\right]\left[\begin{array}{c}
\vdots \\
\mathrm{u}[0] \\
\mathrm{u}[1] \\
\vdots
\end{array}\right],(10
$$

which is equivalent to:

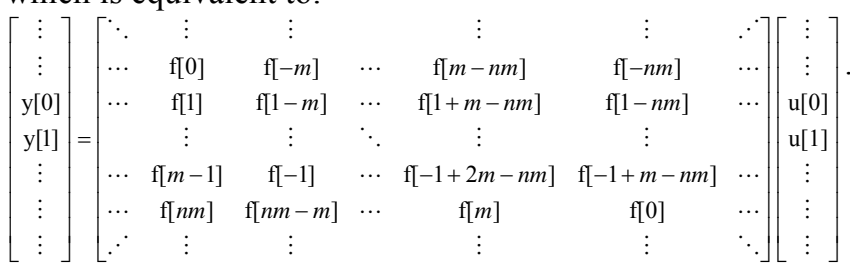

A similar result can be obtained from our second proposed representation. From (11), we can see that the kernel function of our proposed representations is $(m, n)$ shift invariant.

By blocking the input and the output of our proposed representations by $n$ and $m$ samples, respectively, as follows:

$$
\mathbf{u}[k]=[\mathrm{u}[k n], \cdots, \mathrm{u}[k n+n-1]]^{T}, \forall k \in \mathrm{Z},
$$

and

$$
\mathbf{y}[k]=[\mathrm{y}[k m], \quad \cdots, \quad \mathrm{y}[k m+m-1]]^{T}, \forall k \in \mathrm{Z},
$$


where the superscript ${ }^{T}$ denotes the transpose operator, then the blocked output $y[k]$ will shift by 1 sample if the block input $\mathbf{u}[k]$ shifts by 1 sample. From this point of view, we can see that our proposed representations are also $(m, n)$ shift invariant.

\section{PERfECt ReCONSTRUCTION OF INCOMPATIBLE NONUNIFORM FILTER BANKS}

For a given analysis bank with an incompatible set of decimation integers as well as a desirable delay and an overall gain of the whole system (including both the analysis and the synthesis banks), by converting the analysis bank to a uniform filter bank, if the corresponding polyphase matrix is full rank for all frequencies, then there exists a unique synthesis bank that achieves perfect reconstruction. However, in [4] the synthesis bank is realized via linear dual rate systems. By applying the results in this brief, these linear dual rate systems can now be further realized via LTI filters, conventional samplers and block samplers.

For an illustration, consider the example in [4] with the set of decimation integers $\{2,3,6\}$ and the set of analysis filters $H_{0}(z)=1, \quad H_{1}(z)=z^{-4}+z^{-5}$ and $H_{2}(z)=z^{-3}$. Follow the discussion in [4], the synthesis bank involves three linear dual rate systems. The input output relationship of the first linear dual rate system is $y(6 k)=-x(3 k-2), y(6 k+1)=x(3 k-2)$, $y(6 k+2)=0, y(6 k+3)=x(3 k-1), y(6 k+4)=-x(3 k-1)$ and $y(6 k+5)=x(3 k)$; that of the second linear dual rate system is $y(6 k)=x(2 k), \quad y(6 k+1)=0, \quad y(6 k+2)=0, \quad y(6 k+3)=0$, $y(6 k+4)=x(2 k+1)$ and $y(6 k+5)=0$; and that of the third linear dual rate system is $y(6 k)=0, \quad y(6 k+1)=0$, $y(6 k+2)=x(k), \quad y(6 k+3)=0, \quad y(6 k+4)=0 \quad$ and $y(6 k+5)=0$. By using our first proposed realization, the synthesis filters are $F_{0}(z)=z^{-5}+z^{-9}-z^{-10}-z^{-12}+z^{-13}$, $F_{1}(z)=z^{2}+1$, and $F_{2}(z)=z^{-2}$, respectively. The block diagram of the incompatible nonuniform filter bank is shown in Figure $2 \mathrm{a}$. If our second proposed realization is employed, then the synthesis filters are $F_{0}(z)=-z^{-2}+z^{-5}+z^{-10}-z^{-13}+z^{-15}$, $F_{1}(z)=1+z^{-7}$, and $F_{2}(z)=z^{-2}$, respectively. The block diagram of the incompatible nonuniform filter bank is shown in Figure 2b. It is worth noting that the lengths of the corresponding filters in these two proposed representations are the same because $\mathrm{g}[k, l]$ of the corresponding linear dual rate systems in these two proposed representations are the same. However, the time indices of the coefficients of the corresponding filters in these two representations are different because the mappings between $\mathrm{g}[k, l]$ and $\mathrm{f}[k]$ in these two representations are different. Hence, the frequency responses of the corresponding filters in these two representations are different.

There are several points of views for investigations of implementation complexity issues. One is from a software point of view and the other is from a hardware point of view. From a software point of view, the implementation complexities could refer to the operation counts, etc, in which they are dependent on the filter lengths. Form a hardware point of view, the implementation complexities could refer to the number of connections, etc. The number of connections of the proposed representations is directly proportional to the number of channels of the nonuniform filter banks, while that of the nonuniform filter banks realized via linear dual rate systems is directly proportional to the LCM of the set of the decimation integers. Hence, our proposed representations could reduce the number of connections significantly.

For design issues, the design of analysis filters can be formulated as a semi-infinite programming problem with the objective being minimizing the total ripple energy of individual filters subject to continuous constraints defined based on the bounds on the maximum ripple magnitudes. The semi-infinite programming problem can be solved via the dual parameterization method [7]. Once the analysis filters are designed, the synthesis filters could be uniquely derived using our proposed representations and perfect reconstruction can be guaranteed. Since no iteration is required between the design of the analysis bank and the synthesis bank, the convergent problem occurred in traditional design methods based on near perfect reconstruction approaches would not occur.

Consider a design of an incompatible nonuniform filter bank with decimation integers $\{2,3,6\}$. The bandwidth of the filters are $\left[0, \frac{\pi}{2}\right],\left[\frac{\pi}{2}, \frac{5 \pi}{6}\right]$ and $\left[\frac{5 \pi}{6}, \pi\right]$. The transition bandwidth of all the filters is $0.05 \pi$. The specifications on the bounds on the maximum passband and stopband ripple magnitudes of all filters are 0.3 and 0.4 , respectively. The filter length of all filters is 6. By applying our proposed representations on realizing the synthesis bank, the synthesis filters can be derived uniquely and perfect reconstruction is guaranteed.

\section{CONCLUSIONS}

The main contribution of this brief is to represent linear dual rate systems via a series cascade of (i) a conventional expander, an LTI filter and a block decimator, or (ii) a block expander, an LTI filter and a conventional decimator. The difference between our proposed representations and that of the existing one is that an SISO LTI filter is employed in our proposed representations, whereas an LTPV subsystem, a linear switching time varying subsystem or a multiple input multiple output LTI subsystem is required for the existing realizations. These existing representations would expand the input output dimension of the subsystem and cause the complicated design of the circuit layout. On the other hand, our proposed realizations just involve an SISO LTI filter. Hence, the complicated design of the circuit layout does not occur. Consequently, the proposed realizations of linear dual rate systems can thus be employed for perfect reconstruction of incompatible nonuniform filter banks.

\section{APPENDIX A}

To show that $\forall k, l \in \mathrm{Z}, \forall m, n \in \mathrm{Z}^{+}$and $i=0,1, \cdots, m-1$, the 
mapping from $\{0,1, \cdots, m-1\} \times \mathrm{Z}$ to $\mathrm{Z}$, where $[i, l-k n] \in\{0,1, \cdots, m-1\} \times \mathrm{Z}$ and $k m n-m l+i \in \mathrm{Z}$, is bijective, first assume that $\left(i_{1}, z_{1}\right),\left(i_{2}, z_{2}\right) \in\{0,1, \cdots, m-1\} \times Z$ where $\left(i_{1}, z_{1}\right) \neq\left(i_{2}, z_{2}\right)$ and $-m z_{1}+i_{1}=-m z_{2}+i_{2}$. This implies that $m\left(z_{2}-z_{1}\right)=i_{2}-i_{1} . \quad$ Since $\quad i_{1}, i_{2} \in\{0,1, \cdots, m-1\}$, $i_{2}-i_{1} \in[-(m-1),(m-1)]$. As $z_{1}, z_{2} \in Z, m\left(z_{2}-z_{1}\right)=i_{2}-i_{1}$ implies that $i_{1}=i_{2}$ and $z_{1}=z_{2}$. This shows that the mapping is injective. As $\forall y \in Z, \quad \exists i \in\{0,1, \cdots, m-1\}$ and $\exists z \in Z$ such that $i=\bmod (y, m), z=-\frac{y-\bmod (y, m)}{m}$ and $y=-m z+i$. Hence, the mapping is surjective. This proves that the mapping is bijective. This completes the proof.

\section{APPENDIX B}

To show that $\forall l \in Z, \quad \forall m, n \in Z^{+}, \quad k \in\{0,1, \cdots, m-1\}$ and $i \in\{0,1, \cdots, n-1\}$, the mapping from $\{0,1, \cdots, m-1\} \times Z$ to $Z$, where $[k, n l+i] \in\{0,1, \cdots, m-1\} \times \mathrm{Z}$ and $k n-m n l-i \in \mathrm{Z}$, is bijective, first we show that the mapping from $\{0,1, \cdots, n-1\} \times \mathrm{Z}$ to $\mathrm{Z}$, where $[i, l] \in\{0,1, \cdots, n-1\} \times \mathrm{Z}$ and $n l+i \in \mathrm{Z}$, is bijective. Assume that $\left(i_{1}, l_{1}\right),\left(i_{2}, l_{2}\right) \in\{0,1, \cdots, n-1\} \times \mathrm{Z}$ where $\left(i_{1}, l_{1}\right) \neq\left(i_{2}, l_{2}\right)$ and $n l_{1}+i_{1}=n l_{2}+i_{2}$. This implies that $n\left(l_{1}-l_{2}\right)=i_{2}-i_{1}$. Since $i_{1}, i_{2} \in\{0,1, \cdots, n-1\}, \quad i_{2}-i_{1} \in[-(n-1),(n-1)] . \quad$ As $\quad l_{1}, l_{2} \in Z$, $n\left(l_{1}-l_{2}\right)=i_{2}-i_{1}$ implies that $i_{1}=i_{2}$ and $l_{1}=l_{2}$. This shows that the mapping is injective. As $\forall y \in Z, \exists i \in\{0,1, \cdots, n-1\}$ and $\exists l \in \mathrm{Z}$ such that $i=\bmod (y, n), l=\frac{y-\bmod (y, n)}{n}$ and $y=n l+i$. Hence, the mapping is surjective. This proves that the mapping is bijective. Second we show that the mapping from $\{0,1, \cdots, m-1\} \times\{0,1, \cdots, n-1\} \times Z \quad$ to $\mathrm{Z}$, where $[k, i, l] \in\{0,1, \cdots, m-1\} \times\{0,1, \cdots, n-1\} \times \mathrm{Z}$ and $k n-m n l-i \in \mathrm{Z}$, is bijective. Assume $\left(k_{1}, i_{1}, l_{1}\right),\left(k_{2}, i_{2}, l_{2}\right) \in\{0,1, \cdots, m-1\} \times\{0,1, \cdots, n-1\} \times \mathrm{Z} \quad$ where $\left(k_{1}, i_{1}, l_{1}\right) \neq\left(k_{2}, i_{2}, l_{2}\right)$ and $k_{1} n-m n l_{1}-i_{1}=k_{2} n-m n l_{2}-i_{2}$. This implies that $m n\left(l_{2}-l_{1}\right)+n\left(k_{1}-k_{2}\right)+i_{2}-i_{1}=0$. Since $i_{1}, i_{2} \in\{0,1, \cdots, n-1\} \quad$ and $k_{1}, k_{2} \in\{0,1, \cdots, m-1\}$, $k_{1}-k_{2} \in[-(m-1),(m-1)] \quad$ and $\quad i_{1}-i_{2} \in[-(n-1),(n-1)]$. As $m, n \in \mathrm{Z}^{+}, m n\left(l_{2}-l_{1}\right)+n\left(k_{1}-k_{2}\right)+i_{2}-i_{1}=0$ implies that $i_{1}=i_{2}$, $k_{1}=k_{2}$ and $l_{1}=l_{2}$. This shows that the mapping is injective. As $\forall y \in Z, \quad \exists k \in\{0,1, \cdots, m-1\}, \quad \exists i \in\{0,1, \cdots, n-1\}$ and $\exists l \in Z$ such that $i=\left\{\begin{array}{cc}n-\bmod (y, n) & \bmod (y, n) \neq 0 \\ 0 & \bmod (y, n)=0\end{array} \quad k=\frac{\bmod (y+i, m n)}{n} \quad\right.$ and $l=\frac{k n-y-i}{m n}$. Hence, the mapping is surjective. This proves the mapping is bijective. By combining these two results, we can conclude that $\forall l \in Z, \forall m, n \in Z^{+}, k \in\{0,1, \cdots, m-1\}$ and $i \in\{0,1, \cdots, n-1\}$, the mapping from $\{0,1, \cdots, m-1\} \times Z$ to $Z$, where $[k, n l+i] \in\{0,1, \cdots, m-1\} \times \mathrm{Z}$ and $\quad k n-m n l-i \in \mathrm{Z}$, is bijective. This completes the proof.

\section{REFERENCES}

[1] A. Pandharipande and S. Dasgupta, "On biorthgonal nonuniform filter banks and tree structures," IEEE Transactions on Circuits and Systems - I: Fundamental Theory and Applications, vol. 49, no. 10, pp. 1457-1467, 2002.

[2] S. Samadi, M. Omair Ahmad and M. N. S. Swamy, "Characterization of nonuniform perfect-reconstruction filterbanks using unit-step signal," IEEE Transactions on Signal Processing, vol. 52, no. 9, pp. 2490-2499, 2004.

[3] I. Djokovic and P. P. Vaidyanathan, "Results on biorthogonal filter banks," Applied and Computational Harmonic Analysis, vol. 1, pp. 329343, 1994.

[4] T. Chen, L. Qiu and E. W. Bai, "General multirate building structures with application to nonuniform filter banks," IEEE Transactions on Circuits and Systems-II: Analog and Digital Signal Processing, vol. 45, no. 8, pp. 948-958, 1998.

[5] M. R. K. Khansari and A. L. Garcia, "Subband decomposition of signals with generalized sampling," IEEE Transactions on Signal Processing, vol. 41, no. 12, pp. 3365-3376, 1993.

[6] X. G. Xia and B. W. Suter, "Multirate filter banks with block sampling," IEEE Transactions on Signal Processing, vol. 44, no. 3, pp. 484-496, 1996.

[7] C. Y. F. Ho, B. W. K. Ling, Y. Q. Liu, P. K. S. Tam and K. L. Teo, "Optimal design of nonuniform FIR transmultiplexer using semi-infinite programming," IEEE Transactions on Signal Processing, vol. 53, no. 7, pp. 2598-2603, 2005.

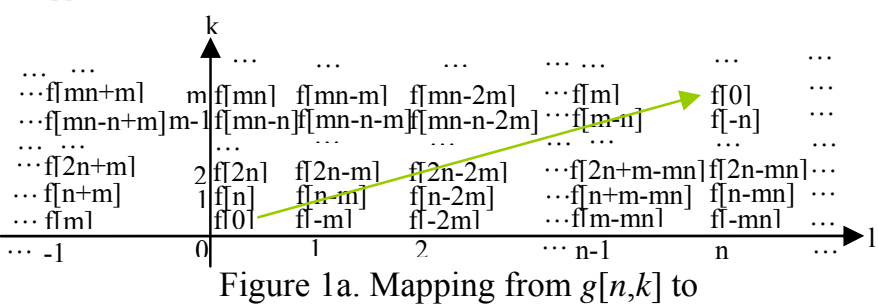
$f[k]$ when $m$ and $n$ are co-prime.

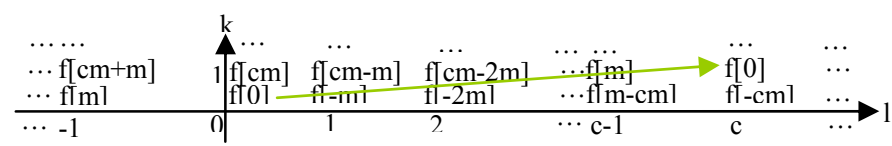

Figure 1b. Mapping from $g[n, k]$ to $f[k]$ when $n=c m$.

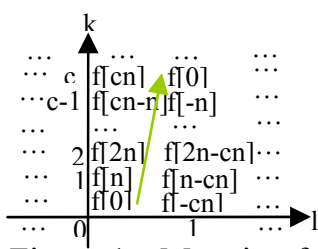

Figure 1c. Mapping from Figure 1d. Mapping from $g[n, k]$ to $f[k]$ when $m=c n$. $g[n, k]$ to $f[k]$ when $m=n$.

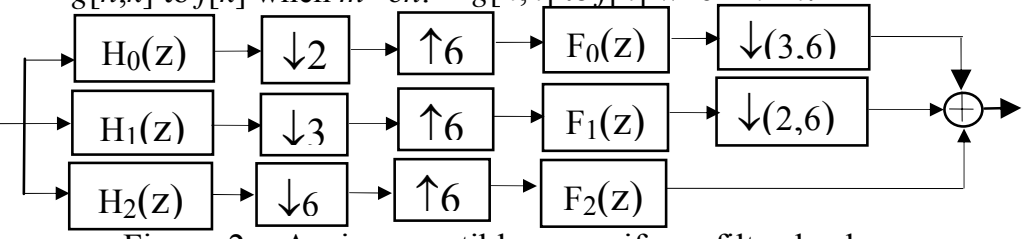

Figure 2a. An incompatible nonuniform filter bank system realized via our first proposed representation.

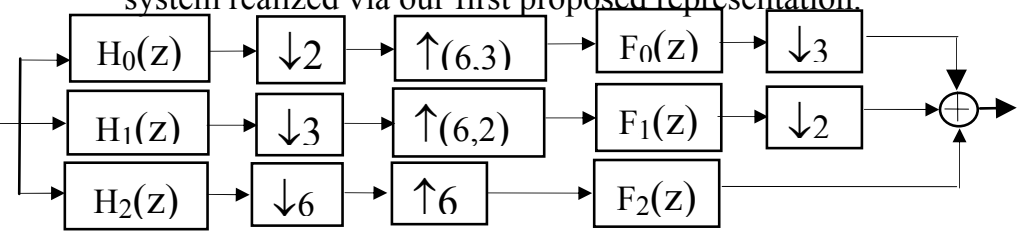

Figure 2b. An incompatible nonuniform filter bank system realized via our second proposed representation. 